\title{
The Richtmyer Memorial Lecture: A Physicist Looks at Mars
}

\author{
ROBERT B. LEIGHTON \\ Division of Physics, Mathematics and Astronomy \\ California Institute of Technology \\ Pasadena, California 91109
}

Response of the Richtmyer Memorial Lecturer to the American Association of Physics Teachers, 1 February 1972.

At this moment there is a Mariner spacecraft circling Mars. Its television cameras have recorded several thousand pictures of that planet and returned them to earth at the rate of several dozen a day. One of the principal goals of the Mariner 9 mission was to learn more about the Martian polar caps - their structure, their seasonal growth and disappearance, their role in Martian weather conditions.

My purpose here is to point out that some of the features of the polar caps and the atmosphere of Mars can apparently be understood very nicely on the basis of elementary physies at a level that should be of interest to teachers of introductory physics courses.

The existence of bright polar caps and dark markings on Mars has been known for more than 200 years. The polar caps were first seen by Christian Huygens in 1668, and by Cassini in the following opposition two years later. Seasonal changes in the polar caps have also long been known on Mars, and are attributable to the fact that the axis of that planet is tilted with respect to its orbital plane, just like earth. As early as 1783 Herschel pointed out that, of all the planets,
Mars seemed more like the Earth than any other. Its period of rotation is, within a few minutes, the same as our own day. The tilt of its axis is about $25^{\circ}$-very close to the $23 \frac{1}{2}^{\circ}$ of the earth.

This idea of an earthlike Mars persisted and grew, culminating in the later part of the nineteenth century and the first part of the present century. The work of Schiaparelli who saw, or thought he saw, linear markings on the planet, and that of Percival Lowell whose pseudoscientific, romantic view of these so called "canali" convinced many people that there was, in fact, a civilization there struggling to preserve its precious supplies of water, dominated Martian studies for several decades. Although these ideas of Mars were contested by other observers, they persisted in the popular domain until very recently.

In 1964 Mariner 4, in flying past the planet, sent back pictures which showed a profusion of impact craters on the surface of the planet. Suddenly, almost overnight, our view of Mars changed from one in which, at least in some people's minds, one could think in terms of vegetation and melting snows, to one of a very desolate, dry, moonlike place.

This moonlike view of Mars persisted for only 4 years, until Mariners 6 and 7 showed further properties of the surface. Mariner 6 showed that, indeed, craters do represent a prevalent topographic form on the planet. Huge craters, some large enough to span from San Diego to Santa Barbara if one of these were superimposed on the California coast, exist and must be a remnant of an extremely ancient surface, perhaps dating from the time of the creation of the planet itself. But also, there is a profusion of small, relatively fresh, round-bottomed craters as contrasted with those large, ancient, very broken, flat-bottomed ones. This double population of craters is of considerable significance because of what it suggests about the history of the surface, and the need for some mechanism, perhaps still in operation, for the removal of some craters. 
But Mariners 6 and 7 also showed that there are other kinds of terrain prevalent on Mars, and Mariner 9 has greatly broadened the spectrum of terrain types. The first of these other characteristic terrains to be seen on the planet is called chaotic terrain. It is characterized by a sunken region, usually sharply bounded by a relatively smooth, lightly cratered plateau, the sunken part consisting of random, hummocky hills, very ancient and worn down in appearance. The name chaotic terrain has been given to features like that on earth, although the Martian analog is much more extensive than anything that has been seen on earth.

Another kind of terrain characteristic of Mars was first recognized in the large circular basin called Hellas. Upon traversing the rim of this basin one goes from a highly cratered terrain outside, into one which shows very few, if any, craters at all. This startling result shows that there must be some very effective erosional processes now at work on Mars, sufficiently active that any craters that are formed in a region like Hellas are quickly erased.

So our view of Mars after Mariners 6 and 7 was that it was beginning to show some characteristic martian features, making it quite unlike either the earth or the moon. And Mariner 9 has added still more to this idea. We now recognize a vast and bewildering variety of topographic forms.

Mars has both an ancient and yet a changing surface: The moon has hardly changed in more than a billion years, whereas, while much of the surface of Mars is similarly ancient, it has present processes at work at various places, at least near its surface.

We have seen, for the first time, clear evidence of endogenic processes on Mars including volcanoes. Indeed, this is not only more conspicuous than on the moon where we have yet to see an earth-like volcano, it may even be more conspicuous than on earth itself. One of the Martian calderas, were it on earth, would be a tourist attraction par excellence! The huge scale of those calderas and the volume of lava erupted vastly overwhelms anything we find on earth.

There are many breaks and fractures in the Martian surface, almost entirely tension effects. That is, they are cracks into which something has slipped or fallen. There is as yet no evidence of compressional deformation which would be expected if the interior of Mars were fully differentiated and there were a convective interior.

There is evidence of flow patterns on the surface, both of a dendritic type and of a sheet flow type. There is evidence of lava flow on the walls of the volcanic calderas. In no case is it clear what flowed. It is easy to assume that flowing water was involved in producing some of the patterns because of our experience on earth. But it would seem wise to reserve judgment. In at least some cases it could have been a fluidized solid.

The polar cap has been found to have a unique morphology seen nowhere else on the planet. We do not know yet what makes the forms we see.

We certainly know much more now about Mars than we have ever known before, but perhaps we understand less about that planet than we thought we understood a few years ago! It is safe to say that Mars has finally emerged from the shadows of fantasy and into the light of rational, direct investigation, and that it is proving itself to be a planet worthy of our curiosity.

But let us return now to the polar caps and consider what they are composed of and how they are related to the atmosphere. The earliest and the most persistent idea of the nature of the polar caps was, of course, that they are composed of ordinary water ice. A few people still talk about the "melting" of the polar caps in discussing seasonal changes. What now seems to be the correct idea was suggested well before 1900 by G. Johnstone Stoney (who suggested the name electron for J. J. Thomson's cathode ray particles). Carbon dioxide had then only recently been liquified and solidified in the laboratory, and he recognized the possibility that Mars, being cooler, might be a place where carbon dioxide could exist in the solid and the gaseous form. He seems to have been quite right; but his idea was ignored, or at least rationalized away, for many decades. With respect to the "melting" of the polar caps, it had also been pointed out that, at the temperature of Mars, one would in any case expect, if water were present, that it could occur only in the form of the vapor and the solid; there would be little if any chance of there being liquid water at any time, anywhere on the surface. 
It has to be said, of course, that the only diagnostic measurement, namely, that of the spectral reflectivity of the polar cap in the near infrared, seems to show an absorption feature characteristic of water ice, and so the most reasonable conclusion, even based on other than romantic evidence, would seem to be that it is water ice. However, a new atmospheric pressure measurement, an actual measurement of the water vapor content, and the Mariner 4 results reopened this question.

Fundamental to the problem of Martian. surface conditions is the question of the composition and surface pressure of the atmosphere. Until 1964 , the surface pressure was thought to be as high as 80 millibars-about $8 \%$ of our own sealevel atmospheric pressure. But now it is known, from ground-based measurements by Kaplan, Münch, and Spinrad, and confirmed by Mariner 4, that the pressure is less than a tenth of that: only 4 or 5 millibars. This gross decrease in the estimated atmospheric pressure has greatly modified our view of the situation on Mars, because of the corresponding conclusion that carbon dioxide, which had been identified as an important constituent some decades earlier by Kuiper, is a major component, rather than a minor component, of the Martian atmosphere. Contrasted with this result are the measurements of water vapor which, after years of careful but fruitless search, were finally successful in showing that water is present, but only to the extent that 20 or 30 precipitable microns, less than a milligram per square centimeter, are present at any given time.

Thus the atmospheric pressure is now thought to be only a few millibars, say 5 millibars, and the atmosphere is predominantly composed of carbon dioxide. Minor amounts of inert gases like nitrogen or argon may be present, and some, but very little, water vapor is also present. The Mariner 4 pictures showed some craters in the higher latitudes which seemed to be outlined in white. This seemed to indicate the presence of frost deposited on the shadowed, interior side of the wall of these craters, and so Bruce Murray and the author ${ }^{1}$ set about to study once again the problem of the heat balance of the planet Mars starting from what, to us, was the beginning. We first considered a thermal model of the planet, in

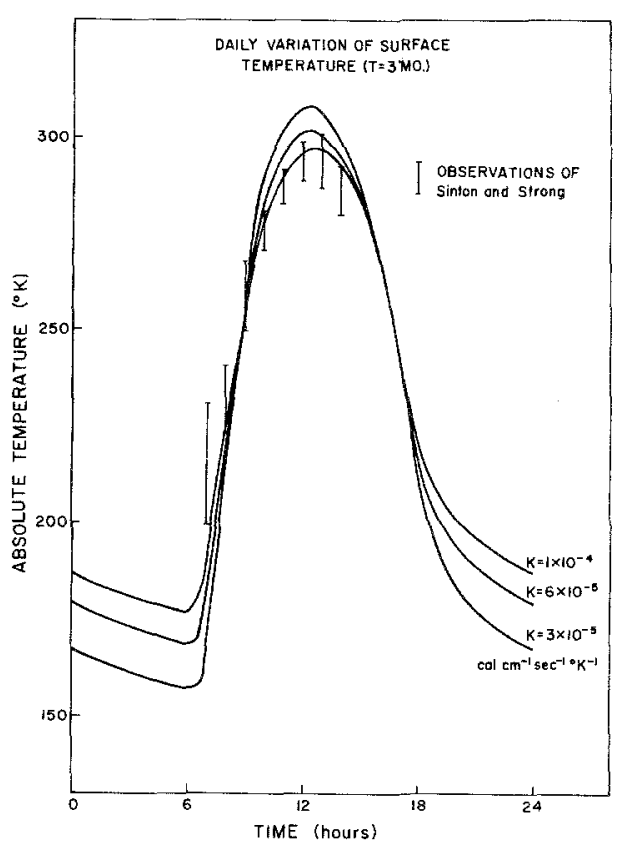

Fig. 1. Daily variation of surface temperature $(T=3 \mathrm{mo})$.

which we assumed the surface to be composed of a very finely powdered silicate-like mineral. We assigned appropriate heat conductivity, specific heat, radiometric albedo, and infrared emissivity to the material. The solar constant is fairly easily calculated to be a little less than half the solar constant on earth.

We first made a computer model in which we considered the absorption of heat into the soil and the radiation of heat away from the planet. During the daytime the sunlight that strikes the surface (with appropriate factors for angle of incidence) is directly reflected, or absorbed to heat up the soil. The surface also radiates in the infrared like a black body. During the night all or part of the heat that was stored in the soil during the daytime would be conducted back to the surface and would radiate into space.

Figure 1 shows the results of the diurnal temperature changes that such a model gives, using various values of the thermal conductivity. We see that one can very easily duplicate, with such a model, the measured values of Sinton and Strong which, being made from earth, only defined the daytime temperatures of the planet. But, having now constructed a physical model of the surface, we could deduce something about 


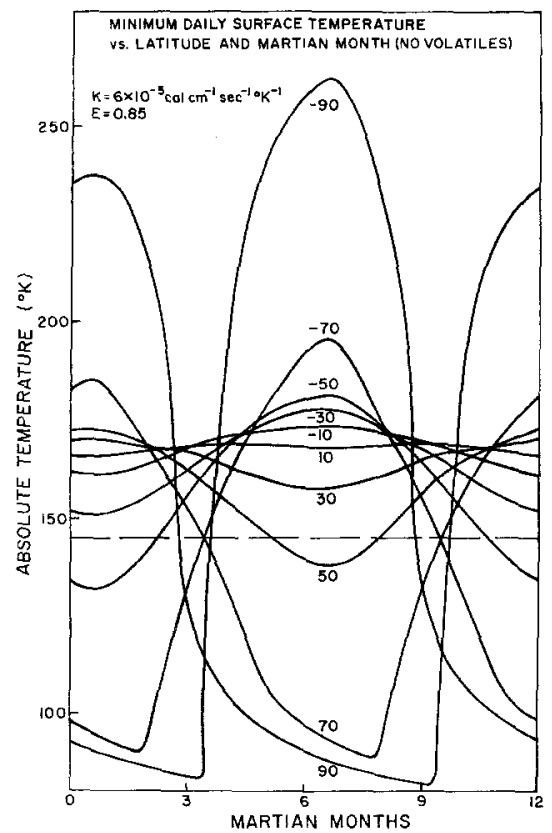

Fig. 2. Minimum daily surface temperature vs latitude and Martian month (no volatiles).

what happened overnight. Near sunset the surface temperature drops rapidly and then more slowly; in the morning when the sun rises, the temperatures goes up very fast because of the very powdery, low-heat-retentivity surface. One can conclude that at night, even at the equator, the surface gets very cold.

We next consider, looking at the 5-millibar pressure of carbon dioxide, what its condensation temperature (the freezing point) would be. It comes out about $145^{\circ} \mathrm{K}$. We then ask whether, if we make the above kind of a heat balance calculation for every part of the planet and throughout the Martian year, there is any part of Mars where the temperature will fall below $145^{\circ} \mathrm{K}$. Figure 2 shows the answer: At latitudes of $50^{\circ}$ or so and poleward, the temperatures fall well below the freezing temperature of carbon dioxide. (Of course, the poles spend 6 Martian months in the dark, and they get exceedingly cold.)

This convinced us very strongly that carbon dioxide would have to condense on Mars. There had been, of course, some speculation about the possible presence of a greenhouse effect which would retard the radiative cooling of the surface. Indeed, the presence of dust or condensation in clouds above the surface would modify the heat balance, but we do not think that such effects are present in sufficient strength to prevent carbon dioxide from condensing.

We next introduced carbon dioxide into our model and studied what happened to that. It dutifully condensed at the north and the south poles. Figure 3 shows the depths of carbon dioxide, in grams per square centimeter, at various latitudes as a function of the Martian calendar date. One sees that polar caps form poleward of $50^{\circ}$ or so in both hemispheres, a little smaller polar cap in the northern hemisphere because of eccentricity of the Mars orbit (the south has a little warmer summer and colder winter than the north does). According to this model, substantial depths, $100 \mathrm{~g} / \mathrm{cm}^{2}$ or more, of carbon dioxide condense. That is a very welcome result in a sense because the water idea, with the existing measurement of only a few microns of precipitable water available in the atmosphere, poses tremendous problems of transport and deposition. It certainly gets cold enough $\left(190^{\circ} \mathrm{K}\right)$ to condense the water, but one faces a diffusion problem: Water is only a minor constituent and is limited in its rate of condensation not by temperature or radiation rate but by a diffusion barrier.

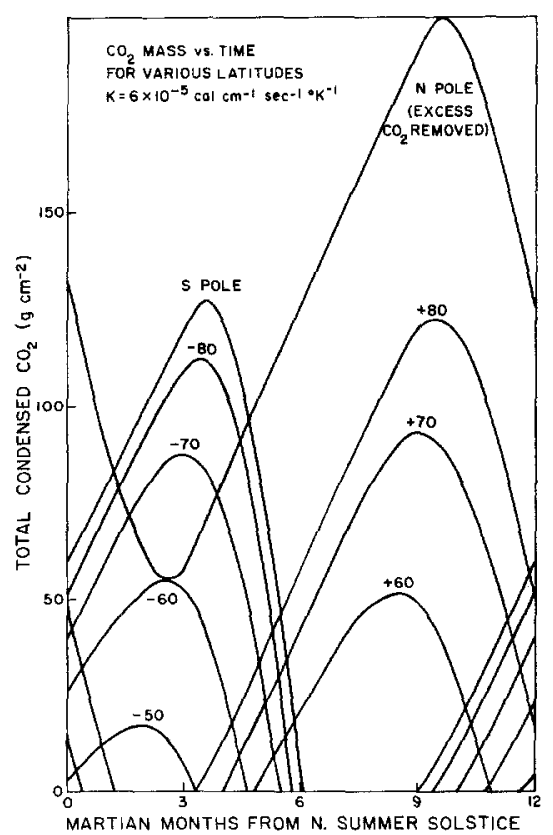

Fig. 3. $\mathrm{CO}_{2}$ mass vs time for various latitudes. 
Carbon dioxide, as the major component, condenses with no such barrier.

Indeed, so much carbon dioxide precipitates on the surface that it should significantly affect the pressure of the Martian atmosphere. Figure 4 shows the modulation of the pressure of the atmosphere that would result from the fact that a substantial part of the atmosphere is lying on the ground part of the time at one pole or the other. Since we can measure the total carbon dioxide content of the Martian atmosphere from Earth, we can actually check up on this feature of the behavior. Such measurements have been made over the past few years, but I have not seen recent results. This modulation is an interesting prediction of the carbon dioxide model, and is a powerful check on its validity.

Now let us consider in a little more detail the behavior of the north polar cap. It is an observed fact, or at least it is written down in books, that the north polar cap never quite disappears, although it gets very small (only $3^{\circ}$ of Martian latitude in radius). (The most recent results from Mariner 9 indicate a small remanent southern cap also.) It didn't strike us at first that the fact that the polar cap becomes very small in area does not necessarily mean that it is then on the verge of disappearing altogether, because that remnant may in fact be quite thick, perhaps hundreds of meters thick, so that it really represents a possibly substantial residuum of carbon dioxide (and water and anything else condensible, since the polar cap is, in effect, a permanent cold trap). That is, it represents the place where any surplus is stored that is not "needed" in the atmosphere.

There is an interesting corollary to this idea: If any part of the residual cap consists of carbon dioxide, it must be isothermal (give or take the little fluctuation of temperature needed to cope with the semiannual modulation of the atmospheric pressure). In that case it is very easy to calculate the temperature $T$ of the polar cap: This must represent a balance between radiant loss of energy at a constant rate ( $\sigma T^{4}$ throughout a Martian year) and the average solar energy absorption by the polar cap throughout the Martian year (there being, by supposition, no other sources of energy as we neglect heat conduction from the interior, atmospheric blanketing, and so forth). Answer: $T=145^{\circ} \mathrm{K}$ !

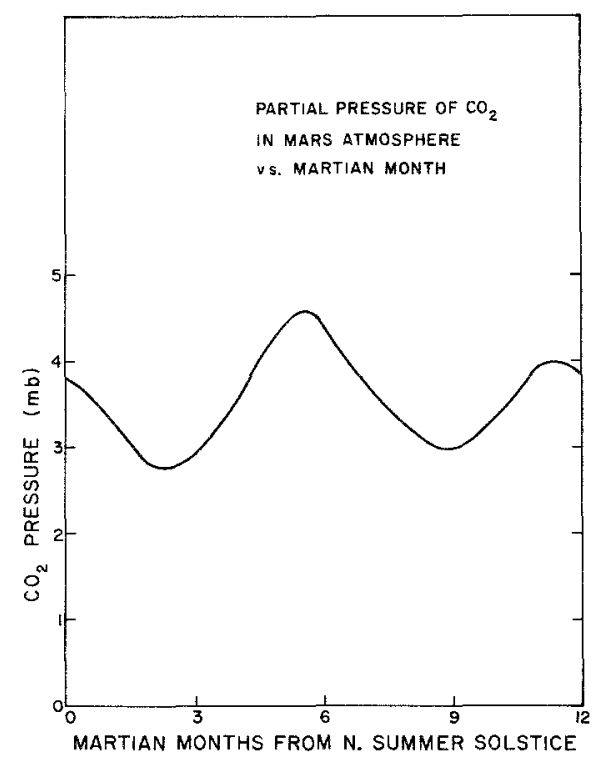

Fig. 4. Partial pressure of $\mathrm{CO}_{2}$ in Martian atmosphere vs Martian month.

Well, we now "erase the blackboard" and start all over. Suppose there were a huge amount of carbon dioxide on Mars; it would reside at the pole, since that is the coldest place. What is the temperature of the polar cap? $145^{\circ} \mathrm{K}$ (we just calculated that). Therefore, what is the pressure of the atmosphere? Answer: the vapor pressure of carbon dioxide at $145^{\circ} \mathrm{K}$. So, Mars is a kind of turned-around planet unlike the earth: It has a cold spot on it which governs the pressure of the atmosphere. Of course, this permanent trap also acts as a trap for water and any other volatiles present. The water whose absorption we do measure is trapped in the polar cap, and may also be trapped elsewhere below layers of dust as permafrost.

The question of how much carbon dioxide is actually present on Mars is, as we see, a point of some interest. Of course, it has to be greater than the 10 to $15 \mathrm{~g} / \mathrm{cm}^{2}$ that corresponds to the known atmospheric pressure on the planet. But, it might be considerably greater than that. Here we at first advanced the idea that the north and the south polar caps would alternate as the repository of any surplus carbon dioxide, in step with the 40 000-year effective precessional cycle on Mars. However, it has recently been shown by Kieffer ${ }^{2}$ that the average insolation at the poles of any 


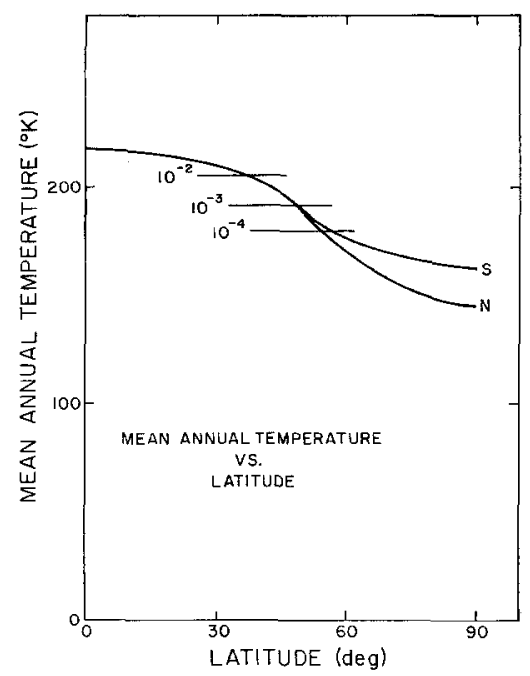

Fig. 5. Mean annual temperature vs latitude.

planet, no matter what its axial tilt, orbital eccentricity, or seasonal date of perihelion, is the same. (This is easy to prove by noticing that the expressions for average polar insolation and average force on the planet, parallel to its rotation axis, are proportional.) Thus the calculation described above should apply equally well to either pole, provided the albedos are similar. It is still possible that there is actually a difference between the two poles and that some long-term transfer of $\mathrm{CO}_{2}$ does take place.

If there is such a difference, there is a limit to how rapidly any excess carbon dioxide could annually be transferred from one pole to the other, perhaps 10 to $20 \mathrm{~g} / \mathrm{cm}^{2}$ per Martian year. If we multiply that by the time for one radian of the 40 thousand year cycle, we come out with about $100 \mathrm{~kg} / \mathrm{cm}^{2}$ as the maximum that could be transported from one pole to the other throughout this precessional cycle. That amount, deposited on the small polar cap, would make it about a kilometer thick, but if we average that over the surface of the planet to find how much $\mathrm{CO}_{2}$ is on Mars, it comes out to be only about 100 $\mathrm{g} / \mathrm{cm}^{2}$; that is, a little less than 10 times the amount that is now in the atmosphere. If, in fact, Mars were born with still more $\mathrm{CO}_{2}$ than that, then there would have to be some residuum of permanent $\mathrm{CO}_{2}$ at both polar caps throughout all time; however, at the "warm" pole, where the surface deposit goes away, there presumably would be some carbon dioxide permafrost left (covered with dust, presumably) throughout this part of the precessional cycle.

Another strong suggestion that comes out of this model is that, over large areas of Mars, there ought to be water ice existing as permafrost just a few centimeters beneath the surface. Figure 5 shows the average annual temperature as a function of latitude. Notice that it is everywhere well below $273^{\circ} \mathrm{K}$. Now the "skin depth" for diurnal temperature variations is only a few centimeters, and even the annual fluctuation is hardly felt more than a meter or so deep. Thus, the mean annual temperature at the surface is also the (constant) temperature a little way beneath the surface. Thus, in the absence of areothermal sources, there should be no liquid ground water at any latitude on Mars. However, poleward of certain latitudes we see that the mean temperature is below the condensation temperature at the indicated masses $\left(\mathrm{g} / \mathrm{cm}^{2}\right)$ of water in the atmosphere, and therefore we expect that water will migrate through the pores of the dusty soil into those cold regions and become trapped as permafrost. Figure 6 shows the depth at which the astronauts will have to dig, as a function of latitude, to find the permafrost. At the north pole, of course, they won't have to dig at all. It's right there on the surface. At the south pole we calculated they would have to dig about $30 \mathrm{~cm}$, but Mariner 9 seems to show us pictures of water ice lying in the open near the south pole as well. More interesting, even at as low a latitude as $60^{\circ}$,

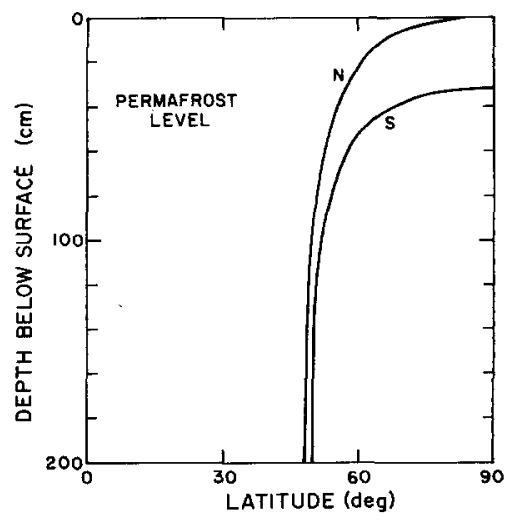

Fig. 6. Permafrost level vs latitude. 
the permafrost should be less than a meter or so down.

The preceding ideas were first advanced seven years ago. While more recent calculations and observational data, particularly that of Mariner 9, have suggested a need for some refinement of the model, the conclusions that the annual polar caps are $\mathrm{CO}_{2}$ ice, that the atmospheric pressure may be determined by the temperature of the coldest spot, probably the north pole, and that water ice permafrost may underlie large areas, seem reasonably secure. In any case, the considerations that lead to these conclusions provide an interesting application of elementary physical mechanisms.

${ }^{1}$ R. B. Leighton and B. C. Murray, Science 153, 3732 (1966).

${ }^{2} \mathrm{H}$. Kieffer, private communication.

\section{A Topical Approach to Astronomy for Nonspecialists}

F. GLENN LIMING, JR.

Department of Physics

Mississippi State University

State College, Mississippi 39762

(Received 16 February 1972)

The goals of a science course for nonscience students can be adequately met with an astronomy course which is also suitable for science majors. The replacement of the survey approach by the topical approach in the course provides greater coherence and meaning for the information presented. A specific set of topics is presented and evaluated in relation to the goals of an astronomy course for nonspecialists.
The thesis of this paper is presented in a sequence of propositions which follows an order of decreasing generality - from widely applicable pedagogical ideas to the specific details of the operation of a particular existing course. The first section proposes a general set of goals for a physical science course for nonscience majors; these goals are related to the needs of the students for whom this type of course is intended. The ideas expressed in this section show the influence of two papers by Crane., ${ }^{1,2}$ The second section demonstrates the desirability of the use of astronomy as the subject of a physical science course for nonscience majors. The reader may refer to an excellent recent paper by Wentzel for additional evidence in support of this opinion. ${ }^{3}$

Most of the astronomy students at Mississippi State University (MSU) have chosen to major in some area of specialization other than the sciences. Many of these students enter the astronomy course as a "captive audience"; 1 they are primarily concerned with "doing their time" in a physical science requirement. However, some of these nonscience students have acquired enough of a true amateur interest in astronomy to be willing to risk their grade averages in a somewhat alien field of learning. They take the course as a free elective rather than as a physical science requirement. Although a more technical course in astronomy has recently been offered to physics majors and other students with experience in introductory physics and calculus, the lower-level astronomy course is still elected by some biolo- 